\title{
Non-Standard Neutrino Interactions from a Triplet Seesaw Model
}

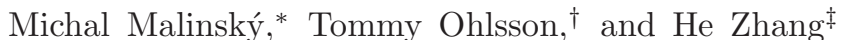 \\ Department of Theoretical Physics, School of Engineering Sciences, \\ Royal Institute of Technology (KTH) - AlbaNova University Center, \\ Roslagstullsbacken 21, 10691 Stockholm, Sweden
}

\begin{abstract}
We investigate non-standard neutrino interactions (NSIs) in the triplet seesaw model featuring non-trivial correlations between NSI parameters and neutrino masses and mixing parameters. We show that sizable NSIs can be generated as a consequence of a nearly degenerate neutrino mass spectrum. Thus, these NSIs could lead to quite significant signals of lepton flavor violating decays such as $\mu^{-} \rightarrow e^{-} \nu_{e} \overline{\nu_{\mu}}$ and $\mu^{+} \rightarrow e^{+} \overline{\nu_{e}} \nu_{\mu}$ at a future neutrino factory, effects adding to the uncertainty in determination of the Earth matter density profile, as well as characteristic patterns of the doubly charged Higgs decays observable at the Large Hadron Collider.
\end{abstract}

\section{INTRODUCTION}

Recently, a large number of studies has been dedicated to the phenomenology of neutrino oscillations and nonstandard neutrino interactions (NSIs) emerging from the effective Lagrangian of the form

$$
\mathcal{L}_{\mathrm{NSI}}=-2 \sqrt{2} G_{F} \varepsilon_{\alpha \beta}^{f f^{\prime} C}\left(\overline{\nu_{\alpha}} \gamma^{\mu} P_{L} \nu_{\beta}\right)\left(\bar{f} \gamma_{\mu} P_{C} f^{\prime}\right),
$$

where $f$ and $f^{\prime}$ denote charged lepton or quark fields, $G_{F}$ is the Fermi coupling constant, and $P_{L}$ and $P_{C}$ (with $C=L, R)$ stand for different chiral projectors. It is generally expected that the effective operator comes out of an underlying theory respecting (or even encompassing) the Standard Model (SM) gauge symmetry upon integrating out a certain set of "heavy" degrees of freedom.

A vast majority of the previous works was concerning the matter-induced NSIs or NSIs at source and/or detector at the level of the effective operator (11). Here, instead, we focus on a particular extension of the SM featuring an extra $S U(2)_{L}$-triplet Higgs [1, 2, 3], which provides a very popular and simple scheme for accommodating Majorana masses of neutrinos within a renormalizable framework, and indeed, can induce significant NSI effects in a future neutrino factory.

This work is organized as follows. In Sec.[I] we present the relations between the effective NSI parameters and the triplet Yukawa couplings. Next, Sec. III] is devoted to a thorough discussion of the experimental constraints stemming namely from exotic charged lepton decays, giving rise to upper bounds for the NSI parameters. Then, in Sec. IV] we provide a simple estimate of the possible effects at a neutrino factory and at the Large Hadron Collider (LHC). Finally, in Sec. V] we summarize our results and conclude.

\footnotetext{
*Electronic address: malinsky@kth.se

$\dagger$ Electronic address: tommy@theophys.kth.se

${ }^{\ddagger}$ Electronic address: zhanghe@kth.se
}

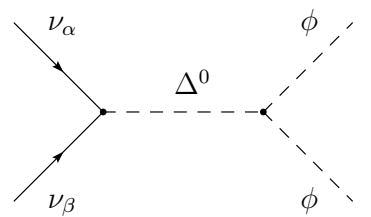

(a)

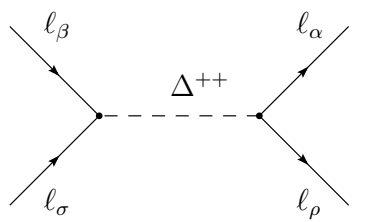

(c)

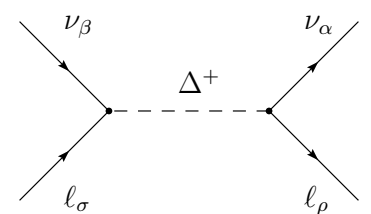

(b)

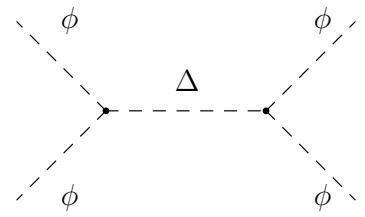

(d)
FIG. 1: Tree level Feynman diagrams with the exchange of heavy triplet fields. The corresponding diagrams are responsible for (a) a light neutrino Majorana mass term, (b) nonstandard neutrino interactions, (c) NSIs of four charged leptons, and (d) self-coupling of the SM Higgs doublet.

\section{LIGHT NEUTRINO MASS MATRIX AND NON-STANDARD INTERACTIONS}

We consider the simple extension of the SM with an extra Higgs triplet [1, 2, 3]. Gauge invariance allows one to add the couplings between the Higgs triplet and two lepton doublets as well as a coupling of the scalar triplet to the SM Higgs doublet [4]

$$
\mathcal{L}_{\Delta}=Y_{\alpha \beta} L_{L}^{T \alpha} C \mathrm{i} \sigma_{2} \Delta L_{L}^{\beta}+\lambda_{\phi} \phi^{T} \mathrm{i} \sigma_{2} \Delta^{\dagger} \phi+\text { H.c. },
$$

where $\Delta$ is a $2 \times 2$ representation of the Higgs triplet field

$$
\Delta=\left(\begin{array}{cc}
\Delta^{+} / \sqrt{2} & \Delta^{++} \\
\Delta^{0} & -\Delta^{+} / \sqrt{2}
\end{array}\right)
$$

and $Y$ is a $3 \times 3$ symmetric matrix in flavor space. Written in component fields, the Yukawa couplings in Eq. (2) receive the form

$$
\begin{aligned}
\mathcal{L}_{Y} & =Y_{\alpha \beta}\left[\Delta^{0} \nu_{\alpha} P_{L} \nu_{\beta}-\frac{1}{\sqrt{2}} \Delta^{+}\left(\overline{\ell_{\alpha}^{c}} P_{L} \nu_{\beta}+\overline{\nu_{\alpha}^{c}} P_{L} \ell_{\beta}\right)\right. \\
& \left.-\Delta^{++} \overline{\ell_{\alpha}^{c}} P_{L} \ell_{\beta}\right]+ \text { H.c. }
\end{aligned}
$$


Integrating out the heavy triplet field at tree level (as illustrated in Fig. 1), one can obtain effective dimension five and six operators, which are responsible for light neutrino masses and NSIs, respectively, [4, 5, [6]

$$
\begin{aligned}
\mathcal{L}_{\nu}^{m} & =\frac{Y_{\alpha \beta} \lambda_{\phi} v^{2}}{m_{\Delta}^{2}}\left(\nu_{L \alpha}^{c} \nu_{L \beta}\right)=-\frac{1}{2}\left(m_{\nu}\right)_{\alpha \beta} \nu_{L \alpha}^{c} \nu_{L \beta} \\
\mathcal{L}_{\mathrm{NSI}} & =\frac{Y_{\sigma \beta} Y_{\alpha \rho}^{\dagger}}{m_{\Delta}^{2}}\left(\overline{\nu_{\alpha}} \gamma_{\mu} P_{L} \nu_{\beta}\right)\left(\overline{\ell_{\rho}} \gamma^{\mu} P_{L} \ell_{\sigma}\right) \\
\mathcal{L}_{4 \ell} & =\frac{Y_{\sigma \beta} Y_{\alpha \rho}^{\dagger}}{m_{\Delta}^{2}}\left(\overline{\ell_{\alpha}} \gamma_{\mu} P_{L} \ell_{\beta}\right)\left(\overline{\ell_{\rho}} \gamma^{\mu} P_{L} \ell_{\sigma}\right)
\end{aligned}
$$

where $v \simeq 174 \mathrm{GeV}$ is the vacuum expectation value of the SM Higgs field. For the NSIs (6) to be potentially sizable, we must require the triplet to be rather light, typically at the $\mathrm{TeV}$ scale. Notice that in such a case the dimensionful parameter $\lambda_{\phi}$ associated with the trilinear Higgs coupling must be small enough to keep the absolute neutrino mass scale proportional to $\lambda_{\phi} v^{2} / m_{\Delta}^{2}$ in the sub-eV range. However, this could be natural, since the symmetry is enhanced in the zero $\lambda_{\phi}$ limit [7].

Comparing Eqs. (5) and (6) with Eq. (1), we can establish relations between the light neutrino mass matrix and NSI parameters in Eq. (11) as (for left lepton chirality)

$$
\varepsilon_{\alpha \beta}^{\rho \sigma}=-\frac{m_{\Delta}^{2}}{8 \sqrt{2} G_{F} v^{4} \lambda_{\phi}^{2}}\left(m_{\nu}\right)_{\sigma \beta}\left(m_{\nu}^{\dagger}\right)_{\alpha \rho} .
$$

Equation (8) clearly shows the correlations between the NSI effects and standard oscillation parameters in the triplet seesaw model. According to Eq. (8), it is easy to see that the NSI parameters are not independent, and in fact, they are strongly tied to the structure of the light neutrino mass matrix $m_{\nu}$. However, not all the $\varepsilon$ 's are physically interesting parameters. For the propagation process in long baseline experiments, neutrinos encounter Earth matter effects and only electron type of NSIs $\varepsilon_{\alpha \beta}^{m} \equiv \varepsilon_{\alpha \beta}^{e e}$ contributes to the matter potential. In addition, Eq. (8) affects neutrino production at neutrino sources, especially for a neutrino factory. More generally, both the processes $\mu^{-} \rightarrow e^{-} \nu_{\mu} \overline{\nu_{\beta}}$ (corresponding to the NSI parameter $\varepsilon_{\mu \beta}^{e \mu}$ ) and $\mu^{-} \rightarrow e^{-} \nu_{\alpha} \overline{\nu_{\beta}}$ (corresponding to $\varepsilon_{\alpha \beta}^{e \mu}$ with $\alpha \neq \mu$ ) may occur, and their contributions have to be added to the SM transition rate either coherently or incoherently, depending on the specific situation. In the following section, we will discuss the current experimental constraints on these NSI parameters in detail.

\section{CONSTRAINTS ON NSI PARAMETERS}

We now summarize the current experimental constraints on NSI parameters defined in Eq. (8). The most stringent experimental bounds come from the lepton family number violating (LFV) decays $\mu \rightarrow 3 e$ and $\tau \rightarrow 3 \ell$, which are tree-level processes induced by $\mathcal{L}_{4 \ell}$ in Eq. (7).

\begin{tabular}{|c|c|c|}
\hline Decay & Constraint on & Bound \\
\hline$\mu^{-} \rightarrow e^{-} e^{+} e^{-}$ & $\left|\varepsilon_{e e}^{e \mu}\right|$ & $3.5 \times 10^{-7}$ \\
\hline$\tau^{-} \rightarrow e^{-} e^{+} e^{-}$ & $\left|\varepsilon_{e e}^{e \tau}\right|$ & $1.6 \times 10^{-4}$ \\
\hline$\tau^{-} \rightarrow \mu^{-} \mu^{+} \mu^{-}$ & $\left|\varepsilon_{\mu \mu}^{\mu \tau}\right|$ & $1.5 \times 10^{-4}$ \\
\hline$\tau^{-} \rightarrow e^{-} \mu^{+} e^{-}$ & $\left|\varepsilon_{e \mu}^{e \tau}\right|$ & $1.2 \times 10^{-4}$ \\
\hline$\tau^{-} \rightarrow \mu^{-} e^{+} \mu^{-}$ & $\left|\varepsilon_{\mu e}^{\mu \tau}\right|$ & $1.3 \times 10^{-4}$ \\
\hline$\tau^{-} \rightarrow e^{-} \mu^{+} \mu^{-}$ & $\left|\varepsilon_{\mu \mu}^{e \tau}\right|$ & $1.2 \times 10^{-4}$ \\
\hline$\tau^{-} \rightarrow e^{-} e^{+} \mu^{-}$ & $\left|\varepsilon_{\mu e}^{e \tau}\right|$ & $9.9 \times 10^{-5}$ \\
\hline$\mu^{-} \rightarrow e^{-} \gamma$ & $\left|\sum_{\alpha} \varepsilon_{\alpha \alpha}^{e \mu}\right|$ & $1.4 \times 10^{-4}$ \\
\hline$\tau^{-} \rightarrow e^{-} \gamma$ & $\left|\sum_{\alpha} \varepsilon_{\alpha \alpha}^{e \tau}\right|$ & $3.2 \times 10^{-2}$ \\
\hline$\tau^{-} \rightarrow \mu^{-} \gamma$ & $\left|\sum_{\alpha} \varepsilon_{\alpha \alpha}^{\mu \tau}\right|$ & $2.5 \times 10^{-2}$ \\
\hline$\mu^{+} e^{-} \rightarrow \mu^{-} e^{+}$ & $\left|\varepsilon_{\mu e}^{\mu e}\right|$ & $3.0 \times 10^{-3}$ \\
\hline
\end{tabular}

TABLE I: Constraints on various $\varepsilon$ 's from $\ell \rightarrow \ell \ell \ell$, one-loop $\ell \rightarrow \ell \gamma$, and $\mu^{+} e^{-} \rightarrow \mu^{-} e^{+}$processes. The experimental bounds have been obtained from Refs. 9, 11].

In terms of NSI parameters, the corresponding decay widths are given by [5]

$$
\begin{aligned}
\Gamma\left(\mu^{-} \rightarrow e^{-} e^{+} e^{-}\right) & =\frac{m_{\mu}^{5}}{24 \pi^{3}} G_{F}^{2}\left|\varepsilon_{e e}^{e \mu}\right|^{2}, \\
\Gamma\left(\tau^{-} \rightarrow \ell_{\alpha}^{-} \ell_{\beta}^{+} \ell_{\alpha}^{-}\right) & =\frac{m_{\tau}^{5}}{24 \pi^{3}} G_{F}^{2}\left|\varepsilon_{\alpha \beta}^{\alpha \tau}\right|^{2}, \\
\Gamma\left(\tau^{-} \rightarrow \ell_{\alpha}^{-} \ell_{\beta}^{+} \ell_{\rho}^{-}\right) & =\frac{m_{\tau}^{5}}{12 \pi^{3}} G_{F}^{2}\left|\varepsilon_{\alpha \beta}^{\rho \tau}\right|^{2} .
\end{aligned}
$$

Another type of stringent constraints on the current model comes from the rare radiative lepton decays $\ell_{\alpha} \rightarrow$ $\ell_{\beta} \gamma$, although these processes emerge at one-loop level. In neglecting the light charged lepton masses, we have [8]

$$
\frac{\Gamma\left(\ell_{\sigma}^{-} \rightarrow \ell_{\rho}^{-} \gamma\right)}{\Gamma\left(\ell_{\sigma}^{-} \rightarrow \ell_{\rho}^{-} \nu_{\sigma} \overline{\nu_{\rho}}\right)}=\frac{\alpha}{6 \pi} \frac{25}{16}\left|\sum_{\alpha} \varepsilon_{\alpha \alpha}^{\rho \sigma}\right|^{2} .
$$

As for the diagonal parts of $Y$, strong bounds come from Bhabha scattering and muonium to antimuonium conversion, which lead to $\left|Y_{e e} Y_{\mu \mu}^{*}\right|<0.1 \times\left(m_{\Delta} / 1 \mathrm{TeV}\right)^{2}$ [9]. There are also constraints from the universality of weak interactions, precise experimental measurements of the $W$ boson mass and the $\rho$ parameter, which are relatively weak compared to the bounds discussed above [10] and will not be elaborated on in our calculations.

The constraints are summarized in Table [1] Notice that the stringent bounds listed in Table \ are related with at least one off-diagonal entry of $m_{\nu}$. In order to receive sizable NSIs effects in neutrino experiments and avoid large LFV processes at the same time, one expects the flavor non-diagonal parts of $m_{\nu}$ to be relatively small. Hence, $m_{\nu}$ should take an approximately diagonal form, which is quite favorable in the case of a nearly degenerate (ND) neutrino mass spectrum $m_{1} \simeq m_{2} \simeq m_{3}$. Then, $m_{\nu}$ approximates to a unit matrix, and the only relevant NSI parameters are $\varepsilon_{e e}^{m}$ and $\varepsilon_{e \mu}^{e \mu}=\left(\varepsilon_{\mu e}^{\mu e}\right)^{*}$. Focusing on the ND case, one can at leading order neglect the neutrino mass-squared differences, so that the generic formula (8) 
is simplified to e.g.

$$
\begin{aligned}
& \varepsilon_{e e}^{m} \simeq-\frac{m_{\Delta}^{2} m_{1}^{2}}{8 \sqrt{2} G_{F} v^{4} \lambda_{\phi}^{2}}\left|\sum_{i=1}^{3} U_{e i}^{2}\right|^{2}, \\
& \varepsilon_{e \mu}^{e \mu} \simeq-\frac{m_{\Delta}^{2} m_{1}^{2}}{8 \sqrt{2} G_{F} v^{4} \lambda_{\phi}^{2}}\left(\sum_{i=1}^{3} U_{e i}^{2}\right)^{*}\left(\sum_{i=1}^{3} U_{\mu i}^{2}\right) .
\end{aligned}
$$

where $U$ is the leptonic mixing matrix. The generic upper bounds on the NSI parameters (8) with respect to the lightest neutrino mass $m_{1}$ are illustrated in Fig. 2, in which a normal neutrino mass hierarchy $m_{1}<m_{2}<m_{3}$ is assumed. We take the triplet Higgs mass $m_{\Delta}=1 \mathrm{TeV}$, which is a typical value within the sensitivity range of the LHC. As for neutrino mixing angles and mass-squared differences, we adopt the values from a global fit given in Ref. [12], and allow all the CP violating phases to range from 0 to $2 \pi$. Similar constraints can be obtained in the case of an inverted neutrino mass hierarchy, since the NSI parameters are only sensitive to $m_{1}$ in the ND region.

Clearly, only $\varepsilon_{e e}^{m}$ and $\varepsilon_{e \mu}^{e \mu}$ are significant at large $m_{1}$ regions, and they are similar in size. The upper bound $\left|\varepsilon_{e \mu}^{e \mu}\right| \sim\left|\varepsilon_{e e}^{m}\right| \lesssim 3 \times 10^{-3}$ can be obtained according to Fig. 2 As for the other NSI parameters, the upper bounds are rather strong, which means that there is no hope for them to be discovered in the near-future long baseline experiments. Thus, $\varepsilon_{e \mu}^{e \mu}$ and $\varepsilon_{e e}^{m}$ are the only relevant quantities to be taken into account in the triplet seesaw model, and we will proceed to investigate their effects at a neutrino factory and at the LHC.

\section{NSI EFFECTS AT A NEUTRINO FACTORY AND AT THE LHC}

The concept of a neutrino factory is proposed to provide the ultimate high-precision measurement of the leptonic mixing parameters and neutrino mass-squared differences. However, the pure and intensive neutrino beams produced in muon decays make such a future facility an ideal place to look for non-standard physics.

a. Wrong sign muons at a near detector: The most striking signal of the underlying triplet model at a neutrino factory corresponds to the processes

$$
\mu^{-} \rightarrow e^{-} \nu_{e} \overline{\nu_{\mu}} \quad \text { and } \quad \mu^{+} \rightarrow e^{+} \overline{\nu_{e}} \nu_{\mu},
$$

leading to would-be observable rates of the "wrong sign" muon tracks in a near detector [13], the so-called zerodistance effect [14]. For instance, a significant admixture of the first process in (15) within the standard muon decay $\mu^{-} \rightarrow e^{-} \nu_{\mu} \overline{\nu_{e}}$ would cause an observable component of $\mu^{+}$to appear along with the "standard" $\nu_{\mu}$-produced muons (in the $\mu^{-}$run) well before the oscillation effects $\overline{\nu_{e}} \rightarrow \overline{\nu_{\mu}}$ set in at longer distances (of the order of a few hundred kilometers). Let us remark that the relative appearance rate of the "wrong sign" muons in a near detector is approximately given by $\left|\varepsilon_{e \mu}^{e \mu}\right|^{2}$ for the $\mu^{-}$run and by $\left|\varepsilon_{\mu e}^{\mu e}\right|^{2}$ for the $\mu^{+}$run, respectively.

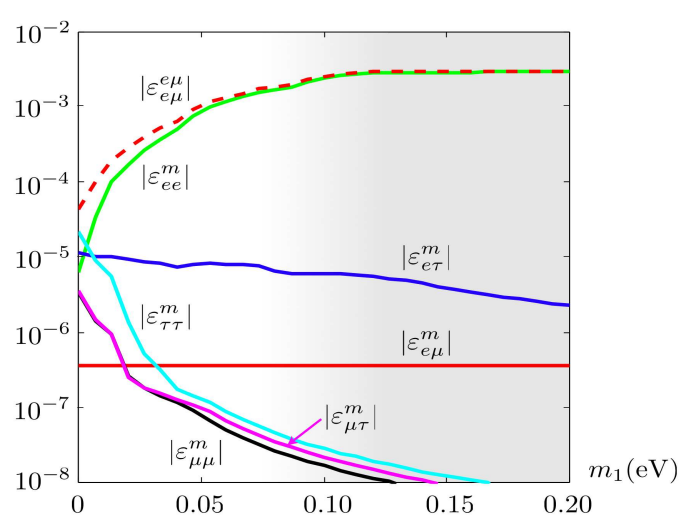

FIG. 2: The upper bounds on the NSI parameters governed by Eq. (8) with respect to the lightest neutrino mass $m_{1}$. Given $m_{1}$, the ratio of $m_{\Delta} / \lambda_{\phi}$ in Eq. (8) (or $\lambda_{\phi}^{-1}$ if $m_{\Delta}$ is fixed) is pushed up unless any of the bounds in Table \ is saturated. The solid curves correspond to constraints on $\left|\varepsilon_{\alpha \beta}^{m}\right|$ parameters, while the dashed curve shows the upper bound on the $\left|\varepsilon_{e \mu}^{e \mu}\right|$ (or $\left.\left|\varepsilon_{\mu e}^{\mu e}\right|\right)$ parameter. Equations (13) and (14) yield the asymptotic values of $\left|\varepsilon_{e \mu}^{e \mu}\right|$ and $\left|\varepsilon_{e e}^{m}\right|$ in the ND region (shaded). The wiggles on the curves are numerical artifacts given by the granularity of the scan over the parameter space.

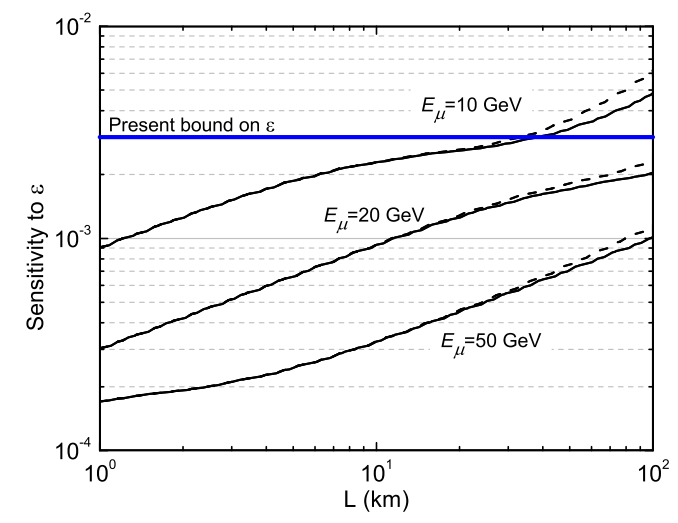

FIG. 3: Sensitivity limits at $90 \%$ C.L. on the parameter $\varepsilon \equiv\left|\varepsilon_{e \mu}^{e \mu}\right|$ as a function of the distance $(L)$ from the source to the near detector. The solid and dashed curves correspond to $\sin ^{2} 2 \theta_{13}=0.01$ and 0.1 , respectively.

In what follows, we illustrate the feasibility of observing such a signal in the near detector of a typical neutrino factory setting. For our numerical simulations, we use the GLoBES software [15, 16] including a standard Abstract Experiment Definition Language (AEDL) file to describe neutrino factory experiments. We assume a setting with approximately $10^{21}$ useful muon decays of each polarity and 4 years of neutrino running plus other 4 years of antineutrino running. A magnetized iron detector with perfect charge identification and a fiducial mass of $1 \mathrm{kt}$ are considered. We show in Fig. 3 the sensitivity to $\varepsilon \equiv\left|\varepsilon_{e \mu}^{e \mu}\right|$ as a function of baseline length for a near detector. The parent muon energies are labeled in the figure. It is obvious that a neutrino factory provides an excellent sensitivity to probe this type of NSI effects. The 
precision of $\varepsilon$ is limited by the baseline (due to the oscillation effects), especially for a large $\theta_{13}$. Thus, a distance $L \lesssim 10 \mathrm{~km}$ would be favorable for the near detector.

Of course, our numerical demonstration in Fig. 3 is only an illustration, and in fact, a detector with fiducial mass $5 \mathrm{kt}$ and $1+1$ years of running also result in similar sensitivities. For the sake of completeness, a more systematic analysis of NSIs at a neutrino factory is desirable. However, since the main purpose of this letter is to clarify the situation about the potentially sizable NSI effects in the triplet seesaw model, a detailed numerical study is beyond the scope of this work and will be elaborated elsewhere.

b. Earth matter density profile uncertainties: In a long baseline experiment, the NSI parameter $\varepsilon_{e e}^{m}$ enters the Hamiltonian as a shift in the Earth matter density, which can be inferred in seismic measurements. Therefore, precision measurements of $\varepsilon_{e e}^{m}$ are related to how well the matter density uncertainty can be constrained. In Ref. [17], with the assumptions of the matter density uncertainty around $1 \%$ and a sizable mixing angle $\sin ^{2} 2 \theta_{13}=0.1$, a sensitivity of the order of a few percent can be achieved in a neutrino factory with a two-detector setup. An improvement by a factor of few would be possible if higher muon energies are considered. Once an accurate geophysical estimate of the Earth matter density uncertainties becomes available, one then may hope to perform a high sensitivity test on $\varepsilon_{e e}^{m}$ in practice.

c. Like-sign di-lepton production at the LHC: It has been pointed out that, in the parameter region $\lambda_{\phi} v^{2} / m_{\Delta}^{2}<10^{-4} \mathrm{GeV}$, the dominant decay channels of the doubly charged Higgs are $\Delta^{ \pm \pm} \rightarrow \ell_{\alpha}^{ \pm} \ell_{\beta}^{ \pm}$(c.f. Ref. [18]), and the partial decay widths are governed by the corresponding triplet Yukawa couplings $\Gamma\left(\Delta^{ \pm \pm} \rightarrow \ell_{\alpha}^{ \pm} \ell_{\beta}^{ \pm}\right) \propto$ $\left|Y_{\alpha \beta}\right|^{2} M_{\Delta}$. According to the analysis above, these widths are correlated to NSI parameters, since both these quantities are sensitive to the neutrino mass spectrum. In the case of sizable NSIs, $\Delta^{ \pm \pm}$produced at the LHC should predominantly decay into a pair of identical leptons. Similarly, one can expect significant NSI effects if the branching ratios of decays to identical leptons $\Delta^{ \pm \pm} \rightarrow \ell_{\alpha}^{ \pm} \ell_{\alpha}^{ \pm}$ would be dramatically larger than for the other channels.

\section{SUMMARY}

We have argued that within the framework of a triplet seesaw model sizable NSIs can naturally emerge as a consequence of a nearly degenerate neutrino mass spectrum. Numerically, upper bounds for the effective couplings like $|\varepsilon| \lesssim 3 \times 10^{-3}$ have been obtained. We have studied in detail the potential of revealing some of these parameters at a near detector of a future neutrino factory and discussed in brief their possible effects on the determination of the Earth matter density profile uncertainties and also collider signatures. We stress that NSI effects are generic features of the seesaw models, and thus should be always properly dealth with. Combined analysis of the electroweak precision tests, the neutrino oscillation experiments, and the future LHC results would be very helpful to figure out the underlying physics behind the neutrino masses and mixing as well as non-standard neutrino interactions.

\section{Acknowledgments}

We are grateful to Walter Winter and Toshihiko Ota for helpful discussions. The work was supported by the Royal Swedish Academy of Sciences (KVA) [T.O.], the Göran Gustafsson Foundation [H.Z.], the Royal Institute of Technology (KTH), contract no. SII-56510 [M.M.], and the Swedish Research Council (Vetenskapsrådet), contract no. 621-2005-3588 [T.O.].
[1] J. Schechter and J. W. F. Valle, Phys. Rev. D22, 2227 (1980).

[2] G. Lazarides, Q. Shafi, and C. Wetterich, Nucl. Phys. B181, 287 (1981).

[3] R. N. Mohapatra and G. Senjanović, Phys. Rev. D23, 165 (1981).

[4] W. Chao and H. Zhang, Phys. Rev. D75, 033003 (2007), hep-ph/0611323.

[5] A. Abada, C. Biggio, F. Bonnet, M. B. Gavela, and T. Hambye, JHEP 12, 061 (2007), arXiv:0707.4058.

[6] M. B. Gavela, D. Hernandez, T. Ota, and W. Winter (2008), arXiv:0809.3451.

[7] G. 't Hooft (1979), lecture given at Cargese Summer Inst., Cargese, France, Aug 26 - Sep 8, 1979.

[8] S. M. Bilenky and S. T. Petcov, Rev. Mod. Phys. 59, 671 (1987).

[9] L. Willmann et al., Phys. Rev. Lett. 82, 49 (1999), hepex/9807011.

[10] S. Antusch, J. P. Baumann, and E. Fernández-Martínez
(2008), arXiv:0807.1003.

[11] C. Amsler et al. (Particle Data Group), Phys. Lett. B667, 1 (2008).

[12] T. Schwetz, M. Tórtola, and J. W. F. Valle (2008), arXiv:0808.2016.

[13] A. Bueno, M. Campanelli, M. Laveder, J. Rico, and A. Rubbia, JHEP 06, 032 (2001), hep-ph/0010308.

[14] P. Langacker and D. London, Phys. Rev. D38, 907 (1988).

[15] P. Huber, M. Lindner, and W. Winter, Comput. Phys. Commun. 167, 195 (2005), hep-ph/0407333.

[16] P. Huber, J. Kopp, M. Lindner, M. Rolinec, and W. Winter, Comput. Phys. Commun. 177, 432 (2007), hep$\mathrm{ph} / 0701187$.

[17] J. Kopp, T. Ota, and W. Winter, Phys. Rev. D78, 053007 (2008), arXiv:0804.2261.

[18] P. Fileviez Perez, T. Han, G.-Y. Huang, T. Li, and K. Wang, Phys. Rev. D78, 015018 (2008), arXiv:0805.3536. 